\title{
Synthesis of hollow spherical tantalum oxide nanoparticles and their photocatalytic activity for hydrogen production
}

\author{
Sen Lin ${ }^{\text {a,* }}$, Lei Shi ${ }^{\mathrm{b}}$, Hisao Yoshida ${ }^{\mathrm{c}}$, Mingrun Li $^{\mathrm{d}}$, Xiaodong Zou ${ }^{\mathrm{d}}$ \\ a Qingdao Institute of Bioenergy and Bioprocess Technology, Chinese Academy of Sciences, 189 Songling Road, Qingdao 266101, China \\ ${ }^{\mathrm{b}}$ Key Laboratory of Fine Chemicals in Universities of Shandong, Shandong Polytechnic University, Daxue Road, Changqing District, Jinan 250353, China \\ ' Department of Applied Chemistry, Graduate School of Engineering, Nagoya University, Furo-cho, Chikusa-ku, Nagoya 464-8603, Japan \\ d Structure Chemistry and Berzelii Centre EXSELENT on Porous Materials, Stockholm University, Stockholm SE-106 91, Sweden
}

\section{A R T I C L E I N F O}

\section{Article history:}

Received 25 June 2012

Received in revised form

15 October 2012

Accepted 18 November 2012

Available online 27 November 2012

Keywords:

Tantalum oxide

Hollow spherical nanoparticle

Synthesis

Photocatalysis

\begin{abstract}
A B S T R A C T
Nano-particles consisting of hollow spherical tantalum oxide (referred to as HSTaO in this work) were synthesized by using the nonionic triblock copolymer F127 as the structure-directing agent in an ethanol aqueous medium. The HSTaO had a large surface area (higher than $100 \mathrm{~m}^{2} / \mathrm{g}$ ), and relatively uniform particle size, in the range of $50-200 \mathrm{~nm}$. The wall thickness of the hollow spherical particles was measured to be about $15-17 \mathrm{~nm}$ and contained ultrafine $\mathrm{Ta}_{2} \mathrm{O}_{5}$ crystallites. The band gap of $\mathrm{HSTaO}$ $(4.3 \mathrm{eV})$ is clearly wider than that of bulk $\mathrm{Ta}_{2} \mathrm{O}_{5}(3.9 \mathrm{eV})$, which can be attributed to the quantum size of the ultrafine $\mathrm{Ta}_{2} \mathrm{O}_{5}$ crystallites. In addition, $\mathrm{HSTaO}$ with core-shell $\mathrm{Ni} / \mathrm{NiO}$ particles (co-catalyst) exhibited similar photocatalytic activity as bulk $\mathrm{Ta}_{2} \mathrm{O}_{5}$ with co-catalyst in hydrogen generation from methanol aqueous solution under photoirradiation, with the HSTaO photocatalyst showing higher stability than the bulk.
\end{abstract}

(c) 2012 Elsevier Inc. All rights reserved.

\section{Introduction}

The photocatalytic splitting of water to generate $\mathrm{H}_{2}$ and $\mathrm{O}_{2}$ as a clean energy source has received extensive attention since the first discovery of $\mathrm{TiO}_{2}$ as a component in the electrochemical photocatalysis of water splitting by Fujishima and Honda [1]. A proper band gap structure is a requirement for water decomposition, and textural properties can also act as an important parameter for the preparation of photocatalysts with excellent photocatalytic properties [2-5]. Generally, the photocatalysis process is taken to include the photogeneration of electron-hole pairs, their migration to external surfaces, and the surface chemical reaction, which are mainly determined by the band gap structure, the crystal structure and quality, and the textural and chemical properties, respectively [2]. In some cases, the morphology and the textural properties can directly affect the photocatalytic properties; for example, some layered structures [6-8] and surface nanostructures [9] enhance hydrogen and oxygen evolution since separate active sites are effective in reducing the reverse reaction.

In recent years, many types of tantalum compounds, such as tantalum oxide, alkali tantalates and alkaline earth tantalates have been recognized as active photocatalysts for water splitting,

\footnotetext{
* Corresponding author. Fax: +8653280662778.

E-mail addresses: linsen@qibebt.ac.cn, leishi78@gmail.com (S. Lin).
}

because of a compatible band gap structure that is formed by the valence band, consisting 02p orbitals, and the conduction band, with $\mathrm{a} \mathrm{d}^{0}$ electronic configuration [9-17]. Recently, Domen et al. synthesized a series of tantalum-based mesoporous materials [18-25] with uniform mesopore structures and large surface areas by using a neutral triblock copolymer Pluronic P123 $\left(\mathrm{EO}_{20} \mathrm{PO}_{70} \mathrm{EO}_{20}\right)$ as the structure-directing agent in a ligand-assisted approach. [26] Some of these novel mesoporous materials exhibited remarkable activity for photocatalytic water decomposition mostly due to their enhanced textural properties. $[18,24]$ The restricted wall thickness provided a large surface area onto which the co-catalyst could be well dispersed, and thus drastically decrease the possibility of recombination of the photogenerated electron-hole pairs in the separation and migration processes. Although mesoporous $\mathrm{Ta}_{2} \mathrm{O}_{5}$ showed higher photocatalytic activity than crystallized $\mathrm{Ta}_{2} \mathrm{O}_{5}$ [18a], the mesoporous structures collapsed to some extent because of the amorphous state of the pore wall structure.

Han and Ying reported the synthesis of nanometer sized mesoporous silica particles with uniform mesoporous structures by using the nonionic triblock copolymer F127 $\left(\mathrm{EO}_{106} \mathrm{PO}_{70} \mathrm{EO}_{106}\right)$ and a cationic fluorocarbon surfactant in $\mathrm{HCl}$ aqueous solution. [27] In order to limit particle growth, weakly acidic synthesis conditions were used to promote a relatively slow silica hydrolysis rate, while at the same time, the silica nanoparticles were surrounded by a fluorocarbon surfactant through the $\mathrm{S}^{+} \mathrm{X}^{-} \mathrm{I}^{+}$ interaction. The particle size of the prepared sample can be restricted to a range of several hundred nanometers. 
Herein, we report the synthesis of hollow sphere-like nanoparticles $\mathrm{Ta}_{2} \mathrm{O}_{5}$ (referred to as "HSTaO" in the following text) in an ethanol aqueous medium by using Pluronic F127 as the template with no additional surfactants. The photocatalytic activity of the sample for hydrogen generation from a methanol aqueous solution under UVlight irradiation was also examined.

\section{Material and methods}

\subsection{Preparation of $\mathrm{HSTaO}$}

The HSTaO sample was prepared using a nonionic Pluronic F127 triblock copolymer $\left(\mathrm{EO}_{106} \mathrm{PO}_{70} \mathrm{EO}_{106}\right)$ (BASF) as the template agent. In a typical synthesis, $2 \mathrm{~g}$ of $\mathrm{F} 127$ was dissolved in $18 \mathrm{~g}$ of ethanol (Kishida Chemical) and stirred vigorously for $30 \mathrm{~min}$. Then, $7.8 \mathrm{~g}$ of tantalum(V)- $n$-butoxide $\left(\mathrm{Ta}\left(\mathrm{OC}_{4} \mathrm{H}_{9}\right)_{5}\right)$ (Mitsuwa Chemical) was added to the mixture and stirred overnight. When distilled water was added to this organic metal complex through a spray with vigorous stirring, hydrolysis occurred immediately to form a white precipitate. The distilled water was poured in gradually up to a total volume of $25 \mathrm{~mL}$. The precipitate was first kept in the supernatant state at room temperature for 6 days, then transferred to a Teflon beaker and aged at $353 \mathrm{~K}$ for 1 day under static conditions, and finally the oven temperature was increased to $373 \mathrm{~K}$ and maintained at this temperature for another day. The product was collected by suction filtration, washed with distilled water and ethanol several times and then dried at $353 \mathrm{~K}$ in air for $12 \mathrm{~h}$. To remove the template by calcination, the product was heated in air from room temperature to $823 \mathrm{~K}$ at a heating rate of $1 \mathrm{~K} / \mathrm{min}$ and then kept at $823 \mathrm{~K}$ for $5 \mathrm{~h}$. In addition to the as-prepared sample, a commercial $\mathrm{Ta}_{2} \mathrm{O}_{5}$ crystal sample (Fluka) was used for comparison without any further treatment.

The co-catalyst nickel species loading was conducted by impregnation of the tantalum oxide sample with a $\mathrm{Ni}\left(\mathrm{NO}_{3}\right)_{2}$ aqueous solution, followed by calcination at $673 \mathrm{~K}$ in air. The nickel-loading amount was $0.1 \mathrm{wt} \%$. For pretreatment, the nickel loaded sample was heated at $673 \mathrm{~K}$ (the lowest temperature for $\mathrm{NiO}$ reduction) for $2 \mathrm{~h}$ in hydrogen atmosphere and then calcined at $473 \mathrm{~K}$ in a flow of oxygen for $1 \mathrm{~h}$ with flow rates of $40 \mathrm{~mL} / \mathrm{min}$ and $50 \mathrm{~mL} / \mathrm{min}$, respectively.

\subsection{Characterization}

Scanning electron microscope (SEM) images were recorded on a JEOL model JSM-6330F field emission scanning electron microscope. Samples for transmission electron microscopy (TEM) observations were crushed and dispersed on porous carbon films on $\mathrm{Cu}$ grids. TEM images were recorded on a JEOL model JEM3010 electron microscope operated at $300 \mathrm{kV}$ with a double tilt $\left( \pm 25^{\circ}\right)$ specimen holder. In order to determine the wall thickness of the HSTaO particles, the magnification was calibrated using TEM images of a $\mathrm{KNb}_{7} \mathrm{O}_{18}$ crystal $(a=b=27.5 \AA, c=3.95 \AA$, Space group: $P 4 / \mathrm{mbm})$ along the $c$-axis taken at the same magnification. X-ray powder diffraction was carried out on a Rigaku model RINT 2100 diffractometer, using $\mathrm{Cu}$ $K_{\alpha}(\lambda=1.54 \AA)$ radiation $(40 \mathrm{kV}, 100 \mathrm{~mA})$. A typical scan speed was $4^{\circ} / \mathrm{min}$ with a step size of $0.02^{\circ}(2 \theta)$ over the range of $10^{\circ}$ to $80^{\circ}$. UV-visible spectra were measured with a JASCO model V-670 spectrophotometer. Powder samples were loaded into a quartz cell with Suprasil windows, and the spectra were collected in the 200$900 \mathrm{~nm}$ wavelength range and were converted from reflection to absorbance by the Kubelka-Munk method. Nitrogen isotherms were determined at $77 \mathrm{~K}$ on a CE Instruments Sorptomatic 1990 using nitrogen (Air Liquide) of 99.999\% purity and helium (Linde) (for dead space calibration). Prior to each experiment, the sample was degassed for $6 \mathrm{~h}$ at $423 \mathrm{~K}$ at a heating rate of $1 \mathrm{~K} / \mathrm{min}$. The specific surface area was evaluated using the Brunauer-Emmett-Teller (BET) method.
Template-removal and crystallization temperatures were measured by differential thermal analysis (DTA) and thermogravimetry (TG) using a Shimadzu model DTG-50 in air with a heating rate of $10 \mathrm{~K} / \mathrm{min}$.

\subsection{Measuement of photocatalytic activity for hydrogen evolution}

Photocatalytic hydrogen generation from a methanol aqueous solution was carried out in an outer irradiation-type reaction vessel made of Pyrex of $120 \mathrm{~mL}$ dead volume with a quartz window. A quantity of $0.1 \mathrm{~g}$ of the photocatalyst powder was dispersed in an aqueous methanol solution ( $5 \mathrm{~g}$ methanol and $45 \mathrm{~g} \mathrm{H}_{2} \mathrm{O}$ ) by a magnetic stirrer. First, the mixture consisting of the photocatalysts suspended in a methanol aqueous solution was treated using ultrasonic irradiation for $0.5 \mathrm{~h}$. Prior to the reactions, the air in the reaction system was completely removed by flowing argon. The light source was a $300 \mathrm{~W}$ Xe illuminator attached with a band-pass filter. The amount of $\mathrm{H}_{2}$ produced was detected by using a gas chromatograph (Shimadzu, model GC-8A, TCD, argon carrier) directly connected to the reaction system. After the reaction, the suspension was filtered, and washed with distilled water and ethanol.

\section{Results and discussion}

\subsection{Structural properties and morphology}

Scanning electron microscope (SEM) images of the bulk $\mathrm{Ta}_{2} \mathrm{O}_{5}$ and the as-prepared HSTaO show different morphology (Fig. 1). The prepared sample displays regular spherical particles of 50-200 nm in size, while the bulk $\mathrm{Ta}_{2} \mathrm{O}_{5}$ shows irregularly shaped blocks of much larger particle size.

TEM images of the as-prepared HSTaO (Fig. 2) shows that a major portion of the material consists of hollow sphere-like nanoparticles with particle sizes ranging from 50 to $200 \mathrm{~nm}$ in diameter. The dark-grey contrast of the particle shells corresponds to tantalum oxide. The light-grey contrast inside the particles corresponds to cavities formed by template-removal at high temperature. In order to check if the particles are spherical, the sample holder was tilted in the TEM around two perpendicular axes and a series of TEM images of the HSTaO nanoparticles was taken at different tilt angles (shown in Fig. 2c). The size and shape of most hollow particles in the images did not change at a different tilt angle, indicating that the particles as well as the cavities are spherical. The wall thickness of the HSTaO particles was estimated to be $15-17 \mathrm{~nm}$, corresponding to around $75 \mathrm{Ta}-\mathrm{O}$ bonds (Fig. 2b). No diffraction spots or polycrystalline rings were observed by TEM, indicating that the walls do not exhibit longrange order. However, as mentioned later, there could be some ultrafine tantalum oxide crystallites inside the walls.

With careful observation, holes were found in the walls of some HSTaO particles (marked in Fig. 2b), which were probably formed during the surfactant decomposition and removal under calcination. Some incomplete particles were also found (Fig. 2a), which were probably broken during calcination or sample preparation for the TEM measurement. These observations support the formation of HSTaO precursors containing surfactant in the core before calcination. Pluronic F127 is well known to form cubic structures of spherical micelles arranged in a close-packing in water [28]. Recently, some hollow silica-based nanospheres with the particle size of 20-200 nm have been synthesized using F127 as template in the aqueous solution by addition of the appropriate inorganic salts, acids or diotin [29-32]. In the synthesis of HSTaO, the spherical micelle aggregations of F127 can form in ethanol. Then, addition of water can lead to an increase in the core radius of micelles. This is due to the more hydrophilic PEO blocks stretch 

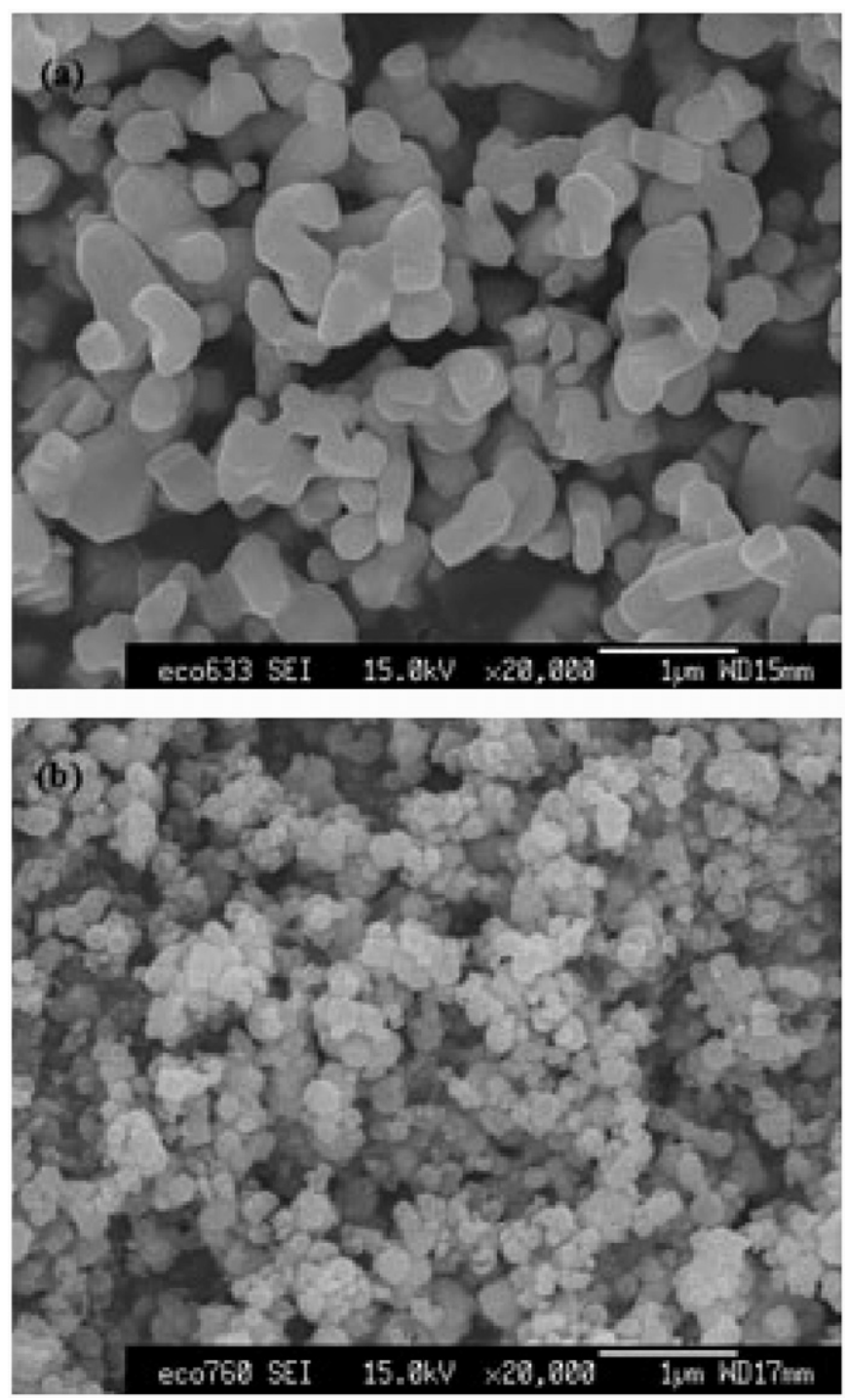

Fig. 1. Scanning electron microscope (SEM) image for (a) bulk $\mathrm{Ta}_{2} \mathrm{O}_{5}$, and (b) prepared sample.

out into the aqueous phase. Plenty of hydroxyl groups generated by the hydrolysis of $\mathrm{Ta}\left(\mathrm{OC}_{4} \mathrm{H}_{9}\right)_{5}$ can simultaneously interact with PEO blocks. Hydrolysis and condensation of $\mathrm{Ta}\left(\mathrm{OC}_{4} \mathrm{H}_{9}\right)_{5}$ took place at the micelle sphere surface, resulting in the fabrication of core/ shell structure. On the other hand, there were some minor small particles that were not hollow but just spherical. This means that preparation conditions may influence the yield of HSTaO particles.

X-ray powder diffraction patterns of a bulk $\mathrm{Ta}_{2} \mathrm{O}_{5}$ and a $\mathrm{HSTaO}$ sample are shown in Fig. 3. For the HSTaO sample, three broad peaks centered at around 27,34 , and $54^{\circ}$ were found, but no diffraction lines assigned to $\mathrm{Ta}_{2} \mathrm{O}_{5}$ were observed. This means that the sample is likely composed of amorphous or very small crystallite domains. Unlike the mesoporous tantalum oxides prepared by the ligand-assisted templating approach reported by Antonelli et al. [26] and Takahara et al. [18], no diffraction peak was observed in the small angle region of $0.5-5^{\circ}$ (not shown here), confirming the absence of regularly ordered meso-pore structures in HSTaO.

The diffuse reflectance spectra of bulk $\mathrm{Ta}_{2} \mathrm{O}_{5}$ and HSTaO are shown in Fig. 4. The absorption edge of HSTaO was located at a shorter wavelength than that of bulk $\mathrm{Ta}_{2} \mathrm{O}_{5}$. The band gaps are roughly estimated to be 4.3 and $3.9 \mathrm{eV}$ for $\mathrm{HSTaO}$ and bulk $\mathrm{Ta}_{2} \mathrm{O}_{5}$, respectively. In the band gap configuration of tantalum oxide, the valence band (VB) consists of O2p orbitals and the conduction band is mainly based on Ta5d orbitals. In a previous study, Kamat and Partric reported a gradual shift to shorter wavelengths of the absorption edge for $\mathrm{ZnO}$ nanosized paricles [33] with size in the region of 2-4 $\mathrm{nm}$ compared to bulk $\mathrm{ZnO}$ powder. Similar results for $\mathrm{CdS}$ and $\mathrm{TiO}_{2}$ were also reported by Wang and Herron [34] and Serpone et al. [35], respectively. Based on the quantum size effect, the distinctly broader band gap of HSTaO compared to that of bulk $\mathrm{Ta}_{2} \mathrm{O}_{5}$ confirms the presence of ultrafine tantalum oxide crystals in the HSTaO wall and the absence of large crystallites of $\mathrm{Ta}_{2} \mathrm{O}_{5}$.

The nitrogen adsorption/desorption isotherms of HSTaO can be approximately assigned to the type II and slit-form hysteresis loop of type $\mathrm{H} 3$ as shown in Fig. 5. Similar to adsorption on a nonporous or macroporous solid, the absence of a plateau in the adsorption branch is indicative of gradual monolayer-multilayer adsorption of the adsorbent on the nonporous solid. Additionally, no clear steep increase was observed in the isotherm, suggesting that the shape of the gap in the wall is slit-form rather than uniform hole-form. Furthermore, the condensation occurring at $P / P^{0} \sim 1$ can be attributed to adsorptive molecules filling the huge pores resulting from the aggregation of the HSTaO particles. On the other hand, H3-type hysteresis loops are assigned to the slitshape pores produced by aggregates of spherical nano-sized particles [36].

The BET specific surface area of the samples before and after $\mathrm{Ni}$ co-catalyst loading were also determined, as given in Table 1 . HSTaO has a high specific surface area of about $100 \mathrm{~m}^{2} / \mathrm{g}$ while bulk $\mathrm{Ta}_{2} \mathrm{O}_{5}$ shows a value as low as $3.2 \mathrm{~m}^{2} / \mathrm{g}$. During the impregnation and heat treatments for co-catalyst loading, the specific surface areas were reduced for both samples, which results from further aggregations of tantalum oxide particles. However, it should be pointed out that bulk $\mathrm{Ta}_{2} \mathrm{O}_{5}$ and $\mathrm{HSTaO}$ showed about 56 and $17 \%$ reductions, respectively. This result indicates that the thermal stability of HSTaO is higher compared to the bulk during the modification process.

Fig. 6 shows the DTA and TG curves of template-containing HSTaO. The weight loss in the relatively low-temperature region $(<473 \mathrm{~K})$ shown in the TG curves can be attributed to the desorption of water and organic molecules from the sample. The TG curves indicate that the template F127 was gradually and completely eliminated in the temperature range 663-823 K. The calculated weight loss in this region was $7.8 \mathrm{wt} \%$, which is significantly less than that of the mesoporous metal-tantalum mixed oxide synthesized in an ethanol solvent by using pluronic P123 as a structure-directing agent [25]. The low weight loss is due to the spherical morphology: unlike the template action of P123, surfactant F127 is favorable for the formation of sphericalform micelles in this synthesis condition rather than the stickform micelles seen in the formation of metal-tantalum mixed oxides. It appears that the low accessibility of oxygen through the small slit-form gaps in the wall of HSTaO during surfactant decomposition makes the practical surfactant decomposition temperature much higher than that of fresh F127 (about $558 \mathrm{~K}$, not shown here). On the other hand, the exothermic peak in the DTA curves at $1003 \mathrm{~K}$ emerged without any weight loss, suggesting that crystallization of tantalum oxides or transformation to other polymorphs [37] occurs in HSTaO around $1000 \mathrm{~K}$.

\subsection{Measuement of photocatalytic activity for hydrogen evolution}

Fig. 7 shows the time course of $\mathrm{H}_{2}$ evolution from a methanol aqueous solution under UV-irradiation with co-catalyst loaded bulk $\mathrm{Ta}_{2} \mathrm{O}_{5}$ and $\mathrm{HSTaO}$ photocatalysts. It was confirmed that both tantalum oxide samples exhibited photocatalytic activity for 

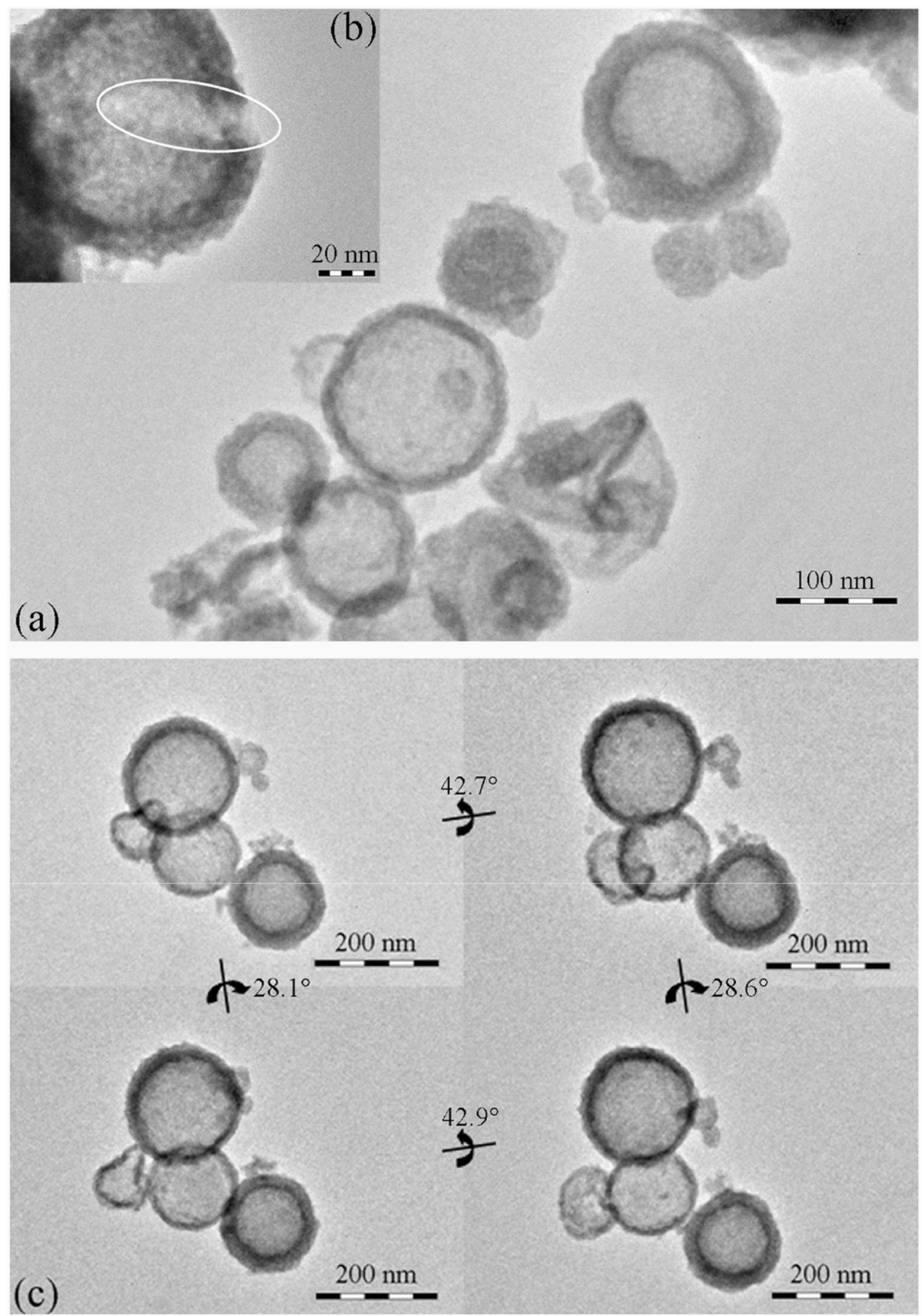

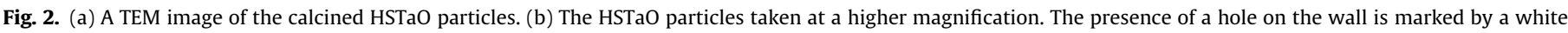

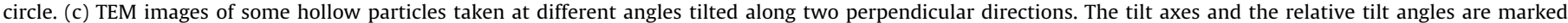
between the images.

hydrogen production from a methanol aqueous solution, meaning that each conduction band had enough potential to reduce protons to hydrogen. Although higher activity was expected for the HSTaO photocatalyst because of the larger specific surface area and the wider band gap, both co-catalyst loaded samples, $(\mathrm{Ni} / \mathrm{NiO}) / \mathrm{Ta}_{2} \mathrm{O}_{5}$ and $(\mathrm{Ni} / \mathrm{NiO}) / \mathrm{HSTaO}$, exhibited almost the same photocatalytic activity with a hydrogen production rate of
112 and $98 \mu \mathrm{mol} \mathrm{h}^{-1}$, respectively, on the first run. The similar hydrogen generation rates suggest that the surface area, morphology and band gap of these two samples does not play a critical role in the photocatalytic reaction under these conditions. Usually, amorphous semiconductor metal oxides tend to exhibit lower photocatalytic activity for hydrogen formation from water solution than a well crystallized semiconductor, a comparison 


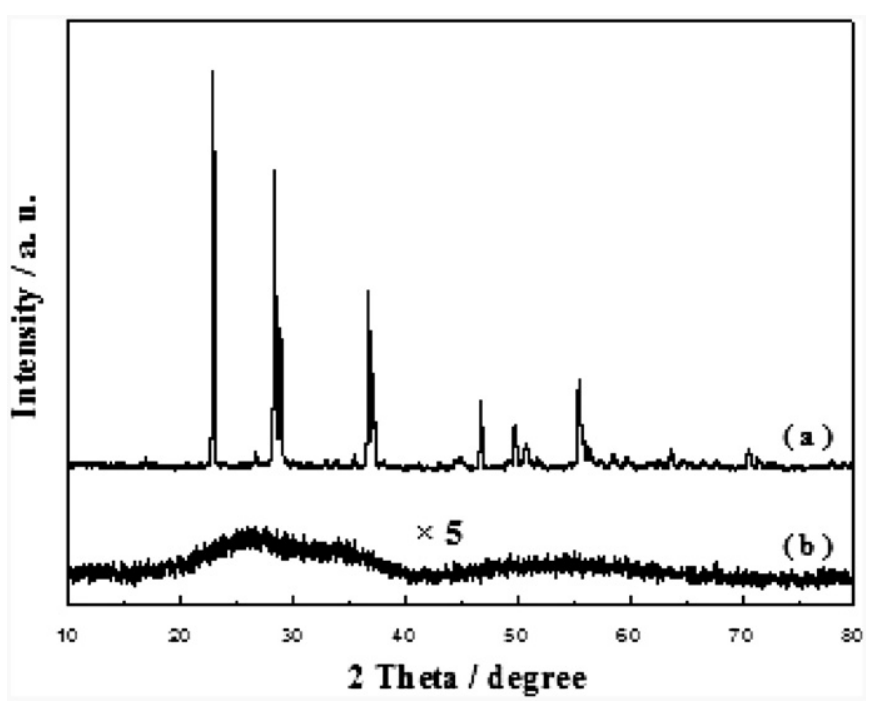

Fig. 3. XRD powder diffraction patterns of (a) bulk $\mathrm{Ta}_{2} \mathrm{O}_{5}$ and (b) HSTaO.

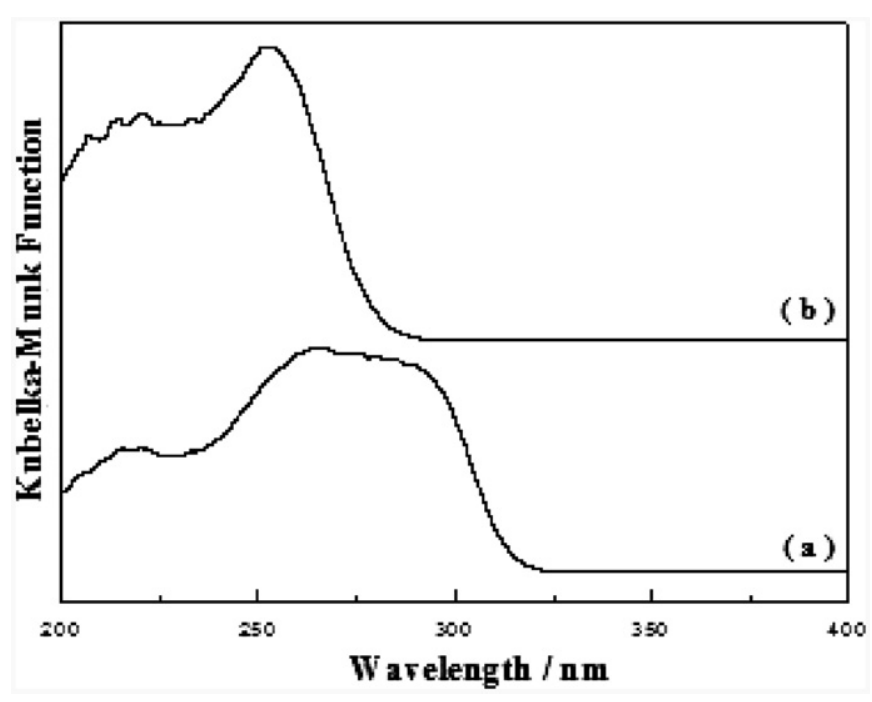

Fig. 4. Diffuse reflectance spectrum of (a) bulk $\mathrm{Ta}_{2} \mathrm{O}_{5}$ and (b) HSTaO.

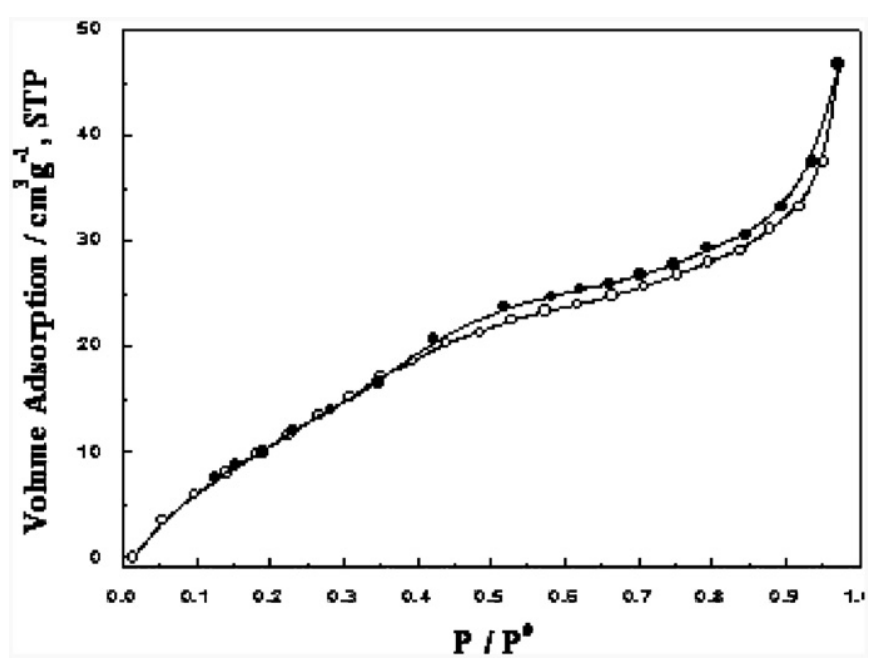

Fig. 5. Nitrogen adsorption/desorption isotherms at $77 \mathrm{~K}$ for template-removal in HSTaO. Open circles are of adsorption and the closed circles are of desorption.
Table 1

Surface area of bulk $\mathrm{Ta}_{2} \mathrm{O}_{5}$ and $\mathrm{HSTaO}$ samples after calcination, nickel-loading and photocatalytic reaction.

\begin{tabular}{lccc}
\hline Sample & \multicolumn{2}{l}{ Surface area $\left(\mathrm{m}^{2} / \mathrm{g}\right)$} & \\
\cline { 2 - 4 } & Calcined sample & Ni loaded sample & After reaction \\
\hline Bulk Ta $2 \mathrm{O}_{5}$ & 3.2 & 1.4 & 0.6 \\
$\mathrm{HSTaO}$ & 101 & 84 & 83 \\
\hline
\end{tabular}

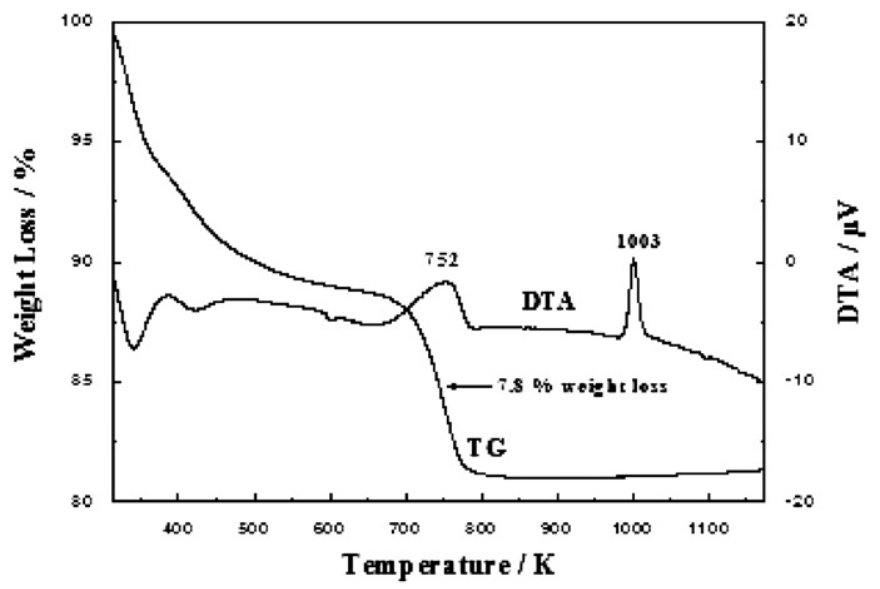

Fig. 6. TG/DTA analysis of template-containing HSTaO. The signal was recorded in the temperature region of $313-1173 \mathrm{~K}$.

that implies the presence of crystallites in the HSTaO photocatalyst. Thus, it is suggested that the wall of HSTaO is composed of ultrafine tantalum oxide crystallites.

When the photocatalytic run was repeated three times, the $\mathrm{H}_{2}$ production rates decreased $\left(112,105\right.$ and $\left.75 \mu \mathrm{mol} \mathrm{h}^{-1}\right)$ for $(\mathrm{Ni} /$ $\mathrm{NiO}) / \mathrm{Ta}_{2} \mathrm{O}_{5}$, but remained almost unchanged $(98,105$ and $109 \mu \mathrm{mol} \mathrm{h}^{-1}$ ) for $(\mathrm{Ni} / \mathrm{NiO}) / \mathrm{HSTaO}$. Deactivation of the bulk $\mathrm{Ta}_{2} \mathrm{O}_{5}$ sample was clearly observed in the third run. This result is possibly due to particle aggregation, a supposition which is supported by the large decrease in specific surface area after the reaction tests, as shown in Table 1 , or else it is due to some change in the co-catalyst (see SI).

Moreover, an induction period was observed for $(\mathrm{Ni} / \mathrm{NiO}) / \mathrm{HSTaO}$, and the period gradually shortened after each run. This result might be due to a change in the oxidation state of the Ni co-catalyst, which could be optimized through photoreduction or photooxidation that occurs in the reaction upon photoirradiation. Most likely, the Ni cocatalysts on HSTaO are highly dispersed as very fine particles, and their oxidation state is easily changed in the induction period, compared to that in bulk $\mathrm{Ta}_{2} \mathrm{O}_{5}$ (Fig. 2 in SI). Further systematic investigation should be carried out to better understand these results.

\section{Conclusion}

Hollow spherical tantalum oxide (HSTaO) nanoparticles were synthesized by using F127 as a structure-directing agent and $\mathrm{Ta}\left(\mathrm{OC}_{4} \mathrm{H}_{9}\right)_{5}$ as a tantalum source in ethanol aqueous solutions. Compared to bulk $\mathrm{Ta}_{2} \mathrm{O}_{5}$ crystals, the HSTaO showed a larger surface area $\left(>100 \mathrm{~m}^{2} / \mathrm{g}\right)$, more uniform spherically shaped particles of $50-200 \mathrm{~nm}$, and a slightly wider band gap $(4.3 \mathrm{eV})$ due to ultrafine $\mathrm{Ta}_{2} \mathrm{O}_{5}$ crystals in the $15-17 \mathrm{~nm}$ thick wall structure (corresponding to at least $75 \mathrm{Ta}-\mathrm{O}$ bonds). Template F127 is favorable for the formation of spherical-form micelles under the synthesis conditions employed in this work, and it can be completely removed at $663-823 \mathrm{~K}$ through the slit-form gaps 




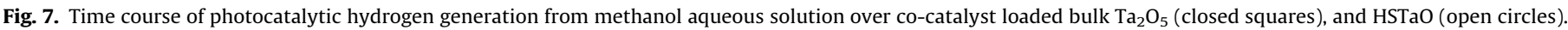
The catalyst weight employed was $0.1 \mathrm{~g}$.

in the wall. After loading nickel species as a co-catalyst and continuously treating it in hydrogen and oxygen, photocatalytic tests were carried out for hydrogen production from a methanol aqueous solution. It was confirmed that $\mathrm{HSTaO}$ showed high photocatalytic activity similar to that of bulk $\mathrm{Ta}_{2} \mathrm{O}_{5}$, and it exhibited higher stability than bulk $\mathrm{Ta}_{2} \mathrm{O}_{5}$ from the first three runs.

\section{Acknowledgment}

The authors gratefully acknowledge the help of Professor M. M. L. Ribeiro Carrott for the $\mathrm{N}_{2}$ sorption measurement and sincerely thank Dr. L. Yuliati, Mr. K. Shimura, Mr. K. Maeda, Mr. H. Yuzawa and Professor R. I. Boughton for the valuable discussion. This study was supported by the Swedish Research Council (VR), the Swedish Governmental Agency for Innovation Systems (VINNOVA) and the Göran-Gustafsson Foundation.

\section{Appendix A. Supporting information}

Supplementary data associated with this article can be found in the online version at http://dx.doi.org/10.1016/j.jssc.2012.11.016.

\section{References}

[1] A. Fujishima, K. Honda, Nature 238 (1972) 37-38.

[2] A. Kudo, H. Kato, I. Tsuji, Chem. Lett. 33 (2004) 1534-1539.

[3] K. Maeda, K. Domen, J. Phys. Chem. C 111 (2007) 7851-7861.

[4] Y. Inoue, Energy Environ. Sci. 2 (2009) 364-386.

[5] A. Kudo, M. Yugo, Chem. Soc. Rev. 38 (2009) 253-278.

[6] A. Kudo, K. Sayama, A. Tanaka, K. Asakura, K. Domen, K. Maruya, T. Onishi, J. Catal. 120 (1989) 337-352.

[7] T. Takata, K. Shinohara, A. Tanaka, M. Hara, J.N. Kondo, K. Domen, J. Photochem. Photobiol., A 106 (1997) 45-49.

[8] K.-ichi Shimizu, S. Itoh, T. Hatamachi, T. Kodama, M. Sato, K. Toda, Chem. Mater. 17 (2005) 5161-5166.
[9] (a) A. Kudo, H. Kato, Chem. Phys. Lett. 331 (2000) 373-377

(b) H. Kato, K. Asakura, A. Kudo, J. Am. Chem. Soc. 125 (2003) 3082-3089.

[10] K. Sayama, H. Arakawa, J. Photochem. Photobiol., A 77 (1994) 243-247.

[11] (a) H. Kato, A. Kudo, Chem. Phys. Lett. 295 (1998) 487-492;

(b) H. Kato, A. Kudo, J. Phys. Chem. B 105 (2001) 4285-4292;

(c) H. Kato, A. Kudo, Catal. Today 78 (2003) 561-569.

[12] H. Kato, A. Kudo, Chem. Lett. (1999) 1207-1208.

[13] T. Ishihara, H. Nishiguchi, K. Fukamachi, Y. Takita, J. Phys. Chem. B 103 (1999) $1-3$.

[14] H.G. Kim, D.W. Hwang, J. Kim, Y.G. Kim, J.S. Lee, Chem. Commun. (1999) 1077-1078.

[15] M. Hara, M. Komoda, H. Hasei, M. Yashima, S. Ikeda, T. Takata, J.N. Kondo, K.J. Domen, Phys. Chem. B 104 (2000) 780-785.

[16] M. Machida, J. Yabunaka, T. Kijima, Chem. Mater. 12 (2000) 812-817.

[17] Z.G. Zou, J.H. Ye, K. Sayama, H. Arakawa, Nature 414 (2001) 625-627.

[18] (a) Y. Takahara, J.N. Kondo, T. Takata, D. Lu, K. Domen, Chem. Mater. 13 (2001) 1194-1199;

(b) J.N. Kondo, Y. Takahara, D. Lu, K. Domen, Chem. Mater. 13 (2001) 1200-1206.

[19] B. Lee, D. Lu, J.N. Kondo, K. Domen, Chem. Commun. (2001) 2118-2119.

[20] B. Lee, D. Lu, J.N. Kondo, K. Domen, Chem. Lett. (2002) 1058-1059.

[21] B. Lee, T. Yamashita, D. Lu, J.N. Kondo, K. Domen, Chem. Mater. 14 (2002) 867-875.

[22] B. Lee, D. Lu, J.N. Kondo, K. Domen, J. Am. Chem. Soc. 124 (2002) $11256-11257$.

[23] D. Lu, G. Hitoki, E. Katou, J.N. Kondo, M. Hara, K. Domen, Chem. Mater. 16 (2004) 1603-1605.

[24] J.N. Kondo, M. Uchida, K. Nakajima, D. Lu, M. Hara, K. Domen, Chem. Mater 16 (2004) 4304-4310

[25] D. Lu, T. Katou, M. Uchida, J.N. Kondo, K. Domen, Chem. Mater. 17 (2005) 632-637.

[26] D.M. Antonelli, J.Y. Ying Chem. Mater. 8 (1996) 874-881.

[27] Y. Han, J.Y. Ying, Angew. Chem. Int. Ed. 44 (2005) 288-292.

[28] R.K. Prud'homme, G.W. Wu, D.K. Schneider, Langmuir 12 (1996) 4651-4659.

[29] J. Liu, S. Bai, H. Zhong, C. Li, Q. Yang, J. Phys. Chem. C 114 (2010) 953-961.

[30] F. Chi, Y.-N. Guo, J. Liu, Y. Liu, Q. Huo, J. Phys. Chem. C 114 (2010) 2519-2523.

[31] M. Mandal, M. Kruk, Chem. Mater. 24 (2012) 123-132.

[32] X.Y. Xiong, L. Guo, Y.C. Gong, Z.L. Li, Y.P. Li, Z.Y. Liu, M. Zhou, Eur. J. Pharm. Sci. 46 (2012) 537-544.

[33] P.V. Kamat, B.J. Partric, J. Phys. Chem. 96 (1992) 6829-6834.

[34] Y. Wang, N. Herron, J. Phys. Chem 95 (1991) 525-532.

[35] N. Serpone, D. Lawless, R. Khairutdinov, E. Pelizzetti, J. Phys. Chem. 99 (1995) $16655-16661$

[36] K.S.W. Sing, D.H. Everett, R.A.W. Haul, L. Moscou, R.A. Pierotti, J. Rouquérol, T. Siemieniewska, Pure Appl. Chem. 57 (1985) 603-619.

[37] C. Askeljung, B.-O. Marinder, M. Sundberg, J. Solid State Chem. 176 (2003) 250-258. 